\title{
ARE NUMBER AND PHASE COMPLEMENTARY OBSERVABLES?
}

\author{
P. BUSCH, P. LAHTI, J.-P. PELLONPÄÄ, AND K. YLINEN
}

\begin{abstract}
We study various ways of characterising the quantum optical number and phase as complementary observables.
\end{abstract}

\section{INTRODUCTION}

The aim of this paper is to clarify the sense in which number and phase in quantum optics can be described as complementary observables. Here phase observables are characterised as phase shift covariant positive operator measures, with the number operator playing the part of the shift generator.

Let $\mathcal{H}$ be a complex separable Hilbert space, $(|n\rangle)_{n \geq 0}$ an orthonormal basis, and $N=\sum_{n=0}^{\infty} n|n\rangle\langle n|$ the associated number observable with the domain $\mathcal{D}(N)=$ $\left\{\left.\varphi \in \mathcal{H}\left|\sum_{n \geq 0} n^{2}\right|\langle\varphi \mid n\rangle\right|^{2}<\infty\right\}$. Let $\mathcal{L}(\mathcal{H})$ denote the set of bounded operators on $\mathcal{H}$, and let $\mathcal{B}([0,2 \pi))$ denote the $\sigma$-algebra of the Borel subsets of the interval $[0,2 \pi)$. We define a phase observable as a positive normalised operator measure $\mathcal{B}([0,2 \pi)) \ni X \mapsto E(X) \in \mathcal{L}(\mathcal{H})$ which is covariant under the shifts generated by the number observable:

$$
e^{i x N} E(X) e^{-i x N}=E(X+x)
$$

for all $X \in \mathcal{B}([0,2 \pi))$ and $x \in[0,2 \pi)$, where the addition $X+x$ is modulo $2 \pi$. The effects $E(X), X \in \mathcal{B}([0,2 \pi))$, are then of the form [1], 2, 3]

$$
E(X)=\sum_{n, m=0}^{\infty} c_{n, m} \frac{1}{2 \pi} \int_{X} e^{i(n-m) x} \mathrm{~d} x|n\rangle\langle m|, X \in \mathcal{B}([0,2 \pi)),
$$

where $\left(c_{n, m}\right)_{n, m \geq 0}$ is the associated phase matrix, that is, a complex matrix generated by a sequence of unit vectors $\left(\xi_{n}\right)_{n \geq 0}$ in $\mathcal{H}: c_{n, m}=\left\langle\xi_{n} \mid \xi_{m}\right\rangle$ for all $n, m \in \mathbb{N}$.

It is well known that among the phase observables there is no projection measure, that is, there is no self-adjoint operator whose spectral measure would be phase shift covariant.

We proceed as follows. In Sec. 2 we present various distinct classes of phase observables, which will provide examples illustrating the degree of commutativity of phase observables in Section 3 and the noncoexistence of number and phase in Section 4. Section 5 reviews the different formalisations of complementarity, which are then applied to number-phase pairs in Sections 6 and 7 . It turns out that only such phase observables $E$, for which $\|E(X)\|=1$ for $E(X) \neq O$, can be complementary to number. In Section 8 we show that the canonical phase as well as the ground state phase space phase (the angle margin of the Husimi $Q$-function) fulfill this necessary 
condition for the number-phase complementarity. Finally, in Section 9 we address the question of the operational content of the notions of complementarity studied here and in earlier work.

\section{EXAMPLES OF PHASE OBSERVABLES}

The canonical phase is defined by the phase matrix whose entries are $c_{n, m}=1$ for all $n, m \geq 0$ :

$$
E_{\text {can }}(X)=\sum_{n, m=0}^{\infty} \frac{1}{2 \pi} \int_{X} e^{i(n-m) x} \mathrm{~d} x|n\rangle\langle m|, \quad X \in \mathcal{B}([0,2 \pi)) .
$$

This is the unique semispectral measure associated with the polar decomposition of the lowering operator, $a=\sum \sqrt{n+1}|n\rangle\langle n+1|=V| a|=V \sqrt{N}, V=\int_{0}^{2 \pi} e^{i x} d E_{\text {can }}(x)$, see, e.g. [4, pp. 141-2], or [2, Example 3.4]. With the choice of the identity matrix as the phase matrix one defines the trivial phase,

$$
E_{\text {triv }}(X)=\frac{1}{2 \pi} \int_{X} \mathrm{~d} x I=\frac{\ell(X)}{2 \pi} I, X \in \mathcal{B}([0,2 \pi)),
$$

where $\ell(X)$ denotes the Lebesgue measure of the Borel set $X$. We define an elementary phase through the equation

$$
E_{\mathrm{el}}(X)=\frac{\ell(X)}{2 \pi} I+w \frac{1}{2 \pi} \int_{X} e^{i(s-t) x} \mathrm{~d} x|s\rangle\left\langle t\left|+\bar{w} \frac{1}{2 \pi} \int_{X} e^{i(t-s) x} \mathrm{~d} x\right| t\right\rangle\langle s|,
$$

where $s \neq t$ and $w$ is any complex number with $|w| \leq 1$. Finally, the matrix elements

$$
c_{n, m}^{|0\rangle}=\frac{\Gamma\left(\frac{n+m}{2}+1\right)}{\sqrt{n !} \sqrt{m !}}, n, m \in \mathbb{N},
$$

constitute the phase space phase observable generated by the ground state $|0\rangle$ :

$$
\begin{aligned}
E_{|0\rangle}(X) & =\sum_{n, m=0}^{\infty} c_{n, m}^{|0\rangle} \frac{1}{2 \pi} \int_{X} e^{i(n-m) x} \mathrm{~d} x|n\rangle\langle m| \\
& =\frac{1}{\pi} \int_{X} \int_{0}^{\infty}\left|r e^{i \theta}\right\rangle\left\langle r e^{i \theta}\right| r \mathrm{~d} r \mathrm{~d} \theta, \quad X \in \mathcal{B}([0,2 \pi)) .
\end{aligned}
$$

We recall that phase observables $E_{1}$ and $E_{2}$ are unitarily equivalent (as phase shift covariant observables) if there is a unitary map $U=\sum_{n=0}^{\infty} e^{i \vartheta_{n}}|n\rangle\langle n|$ such that $E_{2}(X)=U E_{1}(X) U^{-1}$ for all $X \in \mathcal{B}([0,2 \pi))$. A phase observable $E$ is called strong if its $k$-th cyclic moment operator $V_{E}^{(k)}:=\int_{0}^{2 \pi} e^{i k x} \mathrm{~d} E(X)$ is the $k$-th power of its first cyclic moment operator $V_{E}^{(1)}$, that is, if for each $k \geq 0, V_{E}^{(k)}=\left(V_{E}^{(1)}\right)^{k}$. If $E$ is strong, then $\mathbb{N} \ni k \mapsto V_{E}^{(k)} \in \mathcal{L}(\mathcal{H})$ constitutes a (nonunitary) representation of the additive semigroup of the nonnegative integers, and one may ask whether the number observable $n \mapsto|n\rangle\langle n|$ behaves covariantly under the one-sided shifts generated by the phase. Observe that $V_{E}^{(k)}|n+k\rangle=c_{n, n+k}|n\rangle$ for all $n, k \in \mathbb{N}$ which shows that $N$ is $E$-covariant whenever $\left|c_{n, m}\right|=1$ for all $n, m \in \mathbb{N}$.

Combining results from [2, 5] we have the following theorem: 
Theorem 1. For any phase observable $E$, with the phase matrix $\left(c_{n, m}\right)$, the following conditions are equivalent:

(a) $E$ is unitarily equivalent with $E_{\text {can }}$;

(b) $\left|c_{n, m}\right|=1$ for all $n, m$;

(c) E generates the number shifts.

\section{The Degree of COMmutativity of A PhASe OBSERVABle}

Let $\operatorname{com}(E)$ denote the set of vectors $\varphi \in \mathcal{H}$ for which

$$
E(X) E(Y) \varphi=E(Y) E(X) \varphi \text { for all } X, Y \in \mathcal{B}([0,2 \pi)) \text {. }
$$

We say that $E$ is commutative if $\operatorname{com}(E)=\mathcal{H}$, and totally noncommutative if $\operatorname{com}(E)=\{0\}$.

Proposition 2. A phase observable $E$ is commutative if and only if it is the trivial phase $E_{\text {triv }}$.

Proof. The trivial phase $E_{\text {triv }}$ is commutative. Let $E$ be a phase observable with the matrix $\left(c_{n, m}\right)_{n, m \geq 0}$. For any $n \in \mathbb{N}$ and $Y \in \mathcal{B}([0,2 \pi))$, the map

$$
\mathcal{B}([0,2 \pi)) \ni X \mapsto \mu_{n, Y}(X):=\langle n|(E(X) E(Y)-E(Y) E(X))| n\rangle \in \mathbb{C}
$$

is a complex measure. For any $k \in \mathbb{N}$,

$$
\int_{0}^{2 \pi} e^{i k x} \mathrm{~d} \mu_{n, Y}(x)=\left\{\begin{array}{cc}
\left|c_{n, n+k}\right|^{2} \frac{1}{2 \pi} \int_{Y} e^{i k x} \mathrm{~d} x & \text { when } n<k, \\
\left(\left|c_{n, n+k}\right|^{2}-\left|c_{n-k, n}\right|^{2}\right) \frac{1}{2 \pi} \int_{Y} e^{i k x} \mathrm{~d} x & \text { when } n \geq k .
\end{array}\right.
$$

If $E$ is commutative, then $\int_{0}^{2 \pi} e^{i k x} \mathrm{~d} \mu_{n, Y}(x)=0$ for all $Y \in \mathcal{B}([0,2 \pi))$, so that $c_{n, n+k}=0$, for $n<k$, and $\left|c_{n, n+k}\right|=\left|c_{n-k, n}\right|$, for $n \geq k$. Let $n \geq k$, and let $l \geq 0$ be the smallest integer for which $n^{\prime} \equiv n-l k<k$. Then $\left|c_{n, n+k}\right|=\left|c_{n-k, n}\right|=\cdots=$ $\left|c_{n^{\prime}, n^{\prime}+k}\right|=0$. But this means that $c_{n, m}=0$ for all $n \neq m$, that is, $E=E_{\text {triv }}$.

Lemma 3. Let $E$ be a phase observable with the matrix $\left(c_{n, m}\right)_{n, m \geq 0}$. Then

$$
\left\{\varphi \in \mathcal{H} \mid\langle n \mid \varphi\rangle=0 \text { if } c_{n, m} \neq 0 \text { for some } m \neq n\right\} \subseteq \operatorname{com}(E) .
$$

Proof. For the phase observable $E$ with the matrix $\left(c_{n, m}\right)_{n, m \geq 0}$, let

$$
A=\left\{n \in \mathbb{N} \mid c_{n, m}=0 \text { for all } m \neq n\right\},
$$

and define

$$
P_{A}=\sum_{s \in A}|s\rangle\langle s|
$$

Then

and therefore

$$
E(X) P_{A}=\frac{\ell(X)}{2 \pi} P_{A}
$$

$$
E(X) E(Y) P_{A}=\frac{\ell(X) \ell(Y)}{4 \pi^{2}} P_{A}=E(Y) E(X) P_{A}
$$


for all $X, Y \in \mathcal{B}([0,2 \pi))$, so that

$$
\begin{aligned}
P_{A}(\mathcal{H}) & =\left\{\varphi \in \mathcal{H} \mid\langle n \mid \varphi\rangle=0 \text { if } c_{n, m} \neq 0 \text { for some } m \neq n\right\} \\
& \subseteq \operatorname{com}(E) .
\end{aligned}
$$

There are phase observables for which the set inclusion (7) is proper. For instance, the phase observable $E_{w}$, with $c_{0,1}=\overline{c_{1,0}}=c_{1,2}=\overline{c_{2,1}}=w, w \in \mathbb{C}, 0<|w| \leq 1 / \sqrt{2}$, and $c_{n, m}=0$ for all other $n, m \geq 0, n \neq m$, is such.

Proposition 4. Let $E$ be a phase observable with the matrix $\left(c_{n, m}\right)_{n, m \geq 0}$. If $E$ is strong, then

$$
\operatorname{com}(E)=\left\{\varphi \in \mathcal{H} \mid\langle n \mid \varphi\rangle=0 \text { if } c_{n, m} \neq 0 \text { for some } m \neq n\right\} .
$$

Proof. Consider a vector $\psi=\sum_{s=0}^{\infty} d_{s}|s\rangle \in \mathcal{H}$. In view of Lemma B, it remains to be shown that $\psi \in \operatorname{com}(E)$ implies $\psi \in P_{A}(\mathcal{H})$. For any $n \in \mathbb{N}$ and $X, Y \in \mathcal{B}([0,2 \pi))$ we define

$$
F_{n, \psi}(X, Y):=\langle n \mid(E(X) E(Y)-E(Y) E(X)) \psi\rangle \text {. }
$$

For a fixed $Y$, the partial map $X \mapsto F_{n, \psi}(X, Y)$ is a complex measure. For any $k \in \mathbb{N}$,

$$
\begin{aligned}
& \int_{0}^{2 \pi} e^{i k x} \mathrm{~d} F_{n, \psi}(x, Y) \\
& =\sum_{l=0}^{\infty}\left(c_{n, n+k}\langle n+k|E(Y)| l\rangle d_{l}-c_{l, l+k}\langle n|E(Y)| l\rangle d_{l+k}\right) \\
& =: \tilde{F}_{n, k, \psi}(Y) .
\end{aligned}
$$

Again, the map $Y \mapsto \tilde{F}_{n, k, \psi}(Y)$ is a complex measure, and we may carry out the integration

$$
\int_{0}^{2 \pi} e^{i r y} \mathrm{~d} \tilde{F}_{n, k, \psi}(y)=\sum_{l=0}^{\infty}\left(c_{n, n+k} c_{n+k, l} d_{l} \delta_{0, n+k-l+r}-c_{n, l} c_{l, l+k} d_{l+k} \delta_{0, n-l+r}\right)
$$

for all $r \in \mathbb{Z}$. If $\psi \in \operatorname{com}(E)$, then the value of the above integral is zero for all $n, k \in \mathbb{N}, r \in \mathbb{Z}$. This implies

$$
\begin{aligned}
& c_{n, n+k} c_{n+k, n+k+r} d_{n+k+r}=c_{n, n+r} c_{n+r, n+r+k} d_{n+r+k}, \text { when } r \geq-n, \\
& c_{n, n+k} c_{n+k, n+k+r} d_{n+k+r}=0, \text { when }-n>r \geq-n-k .
\end{aligned}
$$

On writing $m=n+k+r$ these conditions are equivalent to:

$$
\begin{aligned}
& c_{n, n+k} c_{n+k, m} d_{m}=c_{n, m-k} c_{m-k, m} d_{m}, \text { when } m \geq k, \\
& c_{n, n+k} c_{n+k, m} d_{m}=0 \text { when, } k>m \geq 0 .
\end{aligned}
$$

It remains to be shown that if $d_{m} \neq 0$, then $c_{n, m}=0$ for all $n \neq m$. Assume, therefore, that $d_{m} \neq 0$ for some $m \in \mathbb{N}$. Then

$$
\begin{aligned}
& c_{n, n+k} c_{n+k, m}=c_{n, m-k} c_{m-k, m}, \text { when } m \geq k, \\
& c_{n, n+k} c_{n+k, m}=0, \text { when } k>m \geq 0 .
\end{aligned}
$$


Putting $n=m$, we get $c_{m, m+k}=0$ for all $k>m$. If $m=0$, then $c_{0, k}=0$ for all $k>0$. Assume next that $m \neq 0$. Since $E$ is strong, we get

$$
c_{m, m+k}=c_{m, m+1} c_{m+1, m+2} \cdots c_{m+k-1, m+k}
$$

for all $k>0$ and

$$
c_{m, m-k}=\overline{c_{m-k, m}}=\overline{c_{m-k, m-k+1} c_{m-k+1, m-k+2}} \cdots \overline{c_{m-1, m}}
$$

for all $k>0, k \leq m$. Further, for $k=1$ and $n=m$, we get from the first of the above equalities that $\left|c_{m-1, m}\right|=\left|c_{m, m+1}\right|$. Thus it suffices to show that $c_{m-1, m}=0$. For that, assume the contrary: $c_{m-1, m} \neq 0$. Fix $l=1,2, \ldots$, and take $n=m+l$, $k=1$. Then

$$
c_{m+l, m+l+1} c_{m+l+1, m}=c_{m+l, m-1} c_{m-1, m} .
$$

Since $E$ is strong, this equation is equivalent to the following:

$$
\left|c_{m+l, m+l+1}\right|^{2} c_{m+l, m}=\left|c_{m-1, m}\right|^{2} c_{m+l, m} .
$$

Therefore, if $c_{m, m+l} \neq 0$, then also $c_{m+l, m+l+1} \neq 0$, and

$$
c_{m, m+l+1}=c_{m, m+l} c_{m+l, m+l+1} \neq 0 .
$$

We have thus shown that $c_{m, m+l} \neq 0$ for all $l=1,2, \ldots$. But this is impossible since, by choosing $n=m$ and $k=m+1$ in (10), we have $c_{m, m+m+1}=0$. Therefore, $c_{m-1, m}=0$.

We note that the equality (8) is not restricted to strong phase observables only. Indeed, an elementary phase with $c_{0,2}=c_{2,0}=1$ is not strong but it has the property (8). The canonical phase and all phase observables unitarily equivalent to it are strong, and all their matrix elements are of modulus one. Therefore, we have:

Corollary 5. The canonical phase, as well as any phase observable unitarily equivalent to it, is totally noncommutative.

\section{The NONCOEXISTENCE OF NUMBER AND PHASE}

The notion of coexistence of observables has been introduced to describe the possibility of measuring the observables together (see, e.g., [6, 7, 8, 9]). If two observable are noncoexistent, they cannot be measured together. Since the number observable $N$ is given by a projection measure $n \mapsto|n\rangle\langle n| \equiv P_{n}$, the coexistence of the number $N$ and a phase $E$ implies their commutativity:

$$
P_{n} E(X)=E(X) P_{n} \quad \text { for all } n \in \mathbb{N}, X \in \mathcal{B}([0,2 \pi)) \text {. }
$$

If we assume that the number $N$ and a phase $E$ commute, then it immediately follows that

$$
E(X)=\sum_{n=0}^{\infty} P_{n} E(X) P_{n}=\sum_{n=0}^{\infty}\langle n|E(X)| n\rangle P_{n}=\frac{\ell(X)}{2 \pi} I
$$

for all $X$, which shows that $E$ is the trivial phase. Hence we have:

Corollary 6. Any nontrivial phase and number are noncoexistent observables. 
Let $\operatorname{com}(N, E)$ denote the set of vectors $\varphi \in \mathcal{H}$ for which

$$
P_{n} E(X) \varphi=E(X) P_{n} \varphi \quad \text { for all } n \in \mathbb{N}, X \in \mathcal{B}([0,2 \pi)) .
$$

Assume that $\varphi \in \operatorname{com}(N, E)$. Then $P_{n} E(X) \varphi=\frac{\ell(X)}{2 \pi} P_{n} \varphi$ for all $n$ and $X$ and thus $E(X) \varphi=\frac{\ell(X)}{2 \pi} \varphi$ for all $X$. Hence, if $\operatorname{com}(N, E)=\mathcal{H}$, then $E$ is the trivial phase. Moreover, if $\varphi \in \operatorname{com}(N, E), \varphi \neq 0$, then $\langle k \mid \varphi\rangle \neq 0$ for some $k$, in which case $c_{n, k}=0$ for all $n \neq k$. Therefore,

$$
\operatorname{com}(N, E)=\left\{\varphi \in \mathcal{H} \mid\langle n \mid \varphi\rangle=0 \text { if } c_{n, m} \neq 0 \text { for some } m \neq n\right\}
$$

When combined with Lemma 3, this shows that

$$
\operatorname{com}(N, E) \subseteq \operatorname{com}(E)
$$

There are examples of phase observables for which this inclusion is a proper one. However, by Proposition 4 , if $E$ is strong, then $\operatorname{com}(N, E)=\operatorname{com}(E)$. In particular, we have:

Proposition 7. For any phase observable $E$, if $c_{n, m} \neq 0$ for all $n, m \geq 0$, then $\operatorname{com}(N, E)=\{0\}$.

Thus, for instance, the phase observables $E_{\text {can }}$ and $E_{|0\rangle}$ commute with $N$ in no state.

We recall that for any $\varphi \in \operatorname{com}(N, E)$, the map

$$
(n, X) \mapsto\left\langle\varphi \mid P_{n} E(X) \varphi\right\rangle=|\langle\varphi \mid n\rangle|^{2} \frac{\ell(X)}{2 \pi}
$$

is a probability bimeasure and thus extends to a joint probability measure of number $N$ and phase $E$ in the vector state $\varphi$, see, e.g. [10, 11].

\section{FORMS OF COMPLEMENTARITY}

The operational idea of complementarity of two observables in the sense of the mutual exclusion of any two experimental procedures permitting the unambiguous definition of these quantities [12 leads in the frame of the quantum theory of measurement [8] to the following condition on number and phase: the number $N$ and a phase $E$ are complementary if for any finite set $\left\{n_{1}, \cdots, n_{k}\right\} \subset \mathbb{N}$ and for any $X \in \mathcal{B}([0,2 \pi))$, for which $O \neq E(X) \neq I$,

$$
\left(\sum_{i=1}^{k} P_{n_{i}}\right) \wedge E(X)=O .
$$

The probabilistic idea of complementarity of two observables in the sense of mutual exclusion of the certain (probability one) predictions of the values of the two observables leads, in the case of number and phase to the following definition: $N$ 
and $E$ are probabilistically complementary if

$$
\begin{aligned}
\sum_{i=1}^{k}\left|\left\langle\varphi \mid n_{i}\right\rangle\right|^{2} & =1 \text { implies that } 0<\langle\varphi \mid E(X) \varphi\rangle<1, \\
\langle\varphi \mid E(X) \varphi\rangle & =1 \text { implies that } 0<\sum_{i=1}^{k}\left|\left\langle\varphi \mid n_{i}\right\rangle\right|^{2}<1,
\end{aligned}
$$

for all vector states $\varphi$, all nonempty sets $\left\{n_{1}, \cdots, n_{k}\right\} \subset \mathbb{N}$, and for any $X \in$ $\mathcal{B}([0,2 \pi))$, such that $O \neq E(X) \neq I$.

There is yet another intuitive notion of complementarity, to which we refer as value complementarity. The idea is that if one of the observables assumes a sharp value, then the other should be uniformly distributed. There are technical problems in formalising this idea because continuous quantities do not have eigenvalues and for unbounded value sets a uniform distribution cannot be easily defined. Here is our proposed definition : $N$ and $E$ are value complementary if the following conditions are satisfied:

(i) for any number eigenstate $|n\rangle$, the phase distribution $X \mapsto\langle n|E(X)| n\rangle$ is uniform;

(ii) for any sequence of vector states $\left(\varphi_{r}\right)$, if the phase distributions $X \mapsto\left\langle\varphi_{r} \mid E(X) \varphi_{r}\right\rangle$ approach a delta distribution centred at some $x_{0} \in[0,2 \pi)$, then the number distributions $n \mapsto\left|\left\langle\varphi_{r} \mid n\right\rangle\right|^{2}$ get increasingly uniform, i.e., $\left|\left\langle\varphi_{r} \mid n\right\rangle\right|^{2} \rightarrow 0$ as $r \rightarrow \infty$.

The probability distribution of any phase observable $E$ is uniform in every number state $|n\rangle$ as $\langle n|E(X)| n\rangle=\frac{\ell(X)}{2 \pi}$. Thus (i) is always fulfilled and the value complementarity of $N$ and $E$ depends only on (ii).

\section{AN EXAMPLE: THE NONCOMPLEMENTARITY OF NUMBER AND ELEMENTARY}

\section{PHASE}

We consider the elementary phase $E_{\mathrm{el}}$ of Equation (5). The spectrum of any $E_{\mathrm{el}}(X) \neq O, I$ consists of three eigenvalues

$$
0 \leq e_{-}(X) \leq e_{0}(X)=\frac{\ell(X)}{2 \pi} \leq e_{+}(X) \leq 1
$$

with

$$
e_{ \pm}(X)=\frac{\ell(X)}{2 \pi} \pm|z|\left|\frac{1}{2 \pi} \int_{X} e^{i(s-t) x} \mathrm{~d} x\right| .
$$

We note that $e_{+}(X)=1$ only when $E_{\mathrm{el}}(X)=I$, and $e_{-}(X)=0$ only when $E_{\mathrm{el}}(X)=$ $O$. Hence we have

$$
\left\|E_{\mathrm{el}}(X)\right\|=e_{+}(X)<1 \text { whenever } E_{\mathrm{el}}(X) \neq I,
$$

and therefore, for any unit vector $\varphi$,

$$
\left\langle\varphi \mid E_{\mathrm{el}}(X) \varphi\right\rangle<1 \text { whenever } E_{\mathrm{el}}(X) \neq I .
$$


We recall that any operator $A$, with $O \leq A \leq I$, can be written in the form $A=\vee(P \wedge A \mid P$ one dimensional projection) [13], where $P \wedge A=\lambda P$, with $\lambda=\|$ $A^{-1 / 2} \varphi \|^{-2}$, for any unit vector $\varphi \in P(\mathcal{H}) \cap \operatorname{ran}\left(A^{1 / 2}\right)$ if the intersection is not the null space, and $\lambda=0$ otherwise. For any $E_{\mathrm{el}}(X) \neq O, I$ we now get

$$
\begin{aligned}
P_{k} \wedge E_{\mathrm{el}}(X) & =\lambda(X) P_{k}, k=s, t, \\
\lambda(X) & =2 \frac{e_{-}(X) e_{+}(X)}{e_{-}(X)+e_{+}(X)} \\
P_{n} \wedge E_{\mathrm{el}}(X) & =\frac{\ell(X)}{2 \pi} P_{n}, n \neq s, t .
\end{aligned}
$$

These relations show that number and elementary phase are not complementary.

For the probabilistic complementarity of $N$ and $E_{\text {el }}$ we need to check only the first implication in the definition (since the other holds trivially due to Eq. (23)). Assume that $\sum_{i=1}^{k}\left|\left\langle\varphi \mid n_{i}\right\rangle\right|^{2}=1$. If $\{s, t\} \not \subset\left\{n_{1}, \cdots, n_{k}\right\}$, then

$$
\left\langle\varphi \mid E_{\mathrm{el}}(X) \varphi\right\rangle=\frac{\ell(X)}{2 \pi},
$$

which is less than 1 whenever $E_{\mathrm{el}}(X) \neq I$. If $s, t \in\left\{n_{1}, \cdots, n_{k}\right\}$, then

$$
\begin{aligned}
\left\langle\varphi \mid E_{\mathrm{el}}(X) \varphi\right\rangle & =\frac{\ell(X)}{2 \pi}+2 \operatorname{Re}\left(z\langle\varphi \mid s\rangle\langle t \mid \varphi\rangle \frac{1}{2 \pi} \int_{X} e^{i(s-t) x} \mathrm{~d} x\right) \\
& \leq \frac{\ell(X)}{2 \pi}+|z| \frac{\ell\left(X^{\prime}\right)}{2 \pi},
\end{aligned}
$$

which is less than 1 whenever $|z|<1$ and $E_{\mathrm{el}}(X) \neq I$. Therefore, $N$ and $E_{\mathrm{el}}$ are probabilistically complementary. In view of (23), they are also value complementary.

\section{Number AND CANONICAL PHASE}

Proposition 8. The canonical phase satisfies $\left\langle\varphi \mid E_{\text {can }}(X) \varphi\right\rangle<1$ for any $X$ such that $E_{\mathrm{can}}(X) \neq I$ and for any unit vector $\varphi \in \mathcal{H}$.

Proof. The minimal Neumark dilation $\tilde{E}_{\text {can }}$ of $E_{\text {can }}$ in $L^{2}([0,2 \pi))$ is the canonical spectral measure $X \mapsto \tilde{E}_{\text {can }}(X)$, with $\tilde{E}_{\text {can }}(X)$ acting as multiplication by the characteristic function $\chi_{X}$. The Hilbert space $\mathcal{H}$ is identified with the Hardy space $H^{2}$ in $L^{2}([0,2 \pi))$. If $\left\langle\varphi \mid E_{\text {can }}(X) \varphi\right\rangle=1$ for some unit vector $\varphi \in H^{2}$ and for some $X$ for which $E_{\text {can }}(X) \neq I$, then $\varphi$ vanishes on the complement set $X^{\prime}$ which has positive measure. It follows from [14, Theorem 13.13] that $\varphi$ is zero, which is a contradiction.

Corollary 9. Number and canonical phase are probabilistically complementary.

We consider next the value complementarity of $N$ and an arbitrary phase $E$.

Proposition 10. Let $\left(\psi_{m}\right)_{m \in \mathbb{N}}$ be a sequence of unit vectors for which the probability measures $X \mapsto\left\langle\psi_{m} \mid E(X) \psi_{m}\right\rangle$ tend (with $m \rightarrow \infty$ ) to a Dirac measure $\delta_{\theta}, \theta \in$ $[0,2 \pi)$. Then the number probabilities $\left|\left\langle\psi_{m} \mid n\right\rangle\right|^{2}$ tend to zero for all $n$. 
Proof. For the phase observable $E$ with the phase matrix $\left(c_{n, m}\right)_{n, m \in \mathbb{N}}$, put $p_{\psi}^{E}(X):=$ $\langle\psi \mid E(X) \psi\rangle$, for any unit vector $\psi$. Let $\left(\psi_{m}\right)_{m \in \mathbb{N}} \subset \mathcal{H}$ be a sequence of unit vectors such that

$$
\lim _{m \rightarrow \infty} p_{\psi_{m}}^{E}([0, x))=\delta_{\theta}([0, x))= \begin{cases}0 & \text { when } 0<x<\theta \\ 1 & \text { when } \theta<x \leq 2 \pi\end{cases}
$$

where $\delta_{\theta}$ is the Dirac measure concentrated on the point $\theta \in[0,2 \pi)$. This implies that $\lim _{m \rightarrow \infty} \int_{0}^{2 \pi} e^{i k x} \mathrm{~d} p_{\psi_{m}}^{E}(x)=e^{i k \theta}$ for all $k \in \mathbb{N}$ (see e.g. [15, Theorem 26.3]). Then

$$
\left|\int_{0}^{2 \pi} e^{i k x} \mathrm{~d} p_{\psi_{m}}^{E}(x)\right|^{2}=\left|\sum_{n=0}^{\infty} c_{n, n+k} \overline{\left\langle n \mid \psi_{m}\right\rangle}\left\langle n+k \mid \psi_{m}\right\rangle\right|^{2} \leq \sum_{l=k}^{\infty}\left|\left\langle l \mid \psi_{m}\right\rangle\right|^{2} \leq 1 .
$$

The left hand side converges to 1 for all $k \in \mathbb{N}$, and so

$$
\sum_{l=k}^{\infty}\left|\left\langle l \mid \psi_{m}\right\rangle\right|^{2} \rightarrow 1 \text { as } m \rightarrow \infty
$$

for all $k \in \mathbb{N}$ if and only if

$$
\sum_{n=0}^{p}\left|\left\langle n \mid \psi_{m}\right\rangle\right|^{2} \rightarrow 0 \text { as } m \rightarrow \infty
$$

for all $p \in \mathbb{N}$.

Equation (24) implies that $\lim _{m \rightarrow \infty}\left\langle\psi_{m} \mid N \psi_{m}\right\rangle \rightarrow \infty$. This situation, where the number gets large and the phase arbitrarily well defined, corresponds to the classical limit for a single mode photon field.

Corollary 11. Number $N$ and any phase $E$ are value complementary.

\section{On THE NORM OF THE PHASE EFFECTS $E(X)$}

In Section 6 we saw that the norm of the effects $E_{\mathrm{el}}(X)$ is strictly less than one whenever $E_{\mathrm{el}}(X) \neq I$. On the other hand, a phase $E$ can be complementary to number only if $\|E(X)\|=1$ for all $E(X) \neq O$. Indeed, assume that $\|E(X)\|<1$ for a nonzero effect $E(X)$. Then the range of $E\left(X^{\prime}\right)$ is $\mathcal{H}$ and therefore $P \wedge E\left(X^{\prime}\right) \neq O$ for any one dimensional projection $P$. Hence $N$ and $E$ are noncomplementary. In this section we show that the norms of any nonzero effects of $E_{\text {can }}$ and $E_{|0\rangle}$ are one. To prove this claim we need to develop some auxiliary results.

To start with we recall (e.g. from [16, p. 138]) that a point $x \in \mathbb{R}$ is a Lebesgue point of a Lebesgue integrable function $f: \mathbb{R} \rightarrow \mathbb{R}$, if

$$
\lim _{r \rightarrow 0_{+}} \frac{1}{2 r} \int_{[x-r, x+r]}|f(y)-f(x)| d y=0 .
$$

We only need this notion in the case where $f$ is the characteristic function $\chi_{X}$ of a Borel $X$. Clearly, $x \in X$ is a Lebesgue point of $\chi_{X}$ if and only if

$$
\lim _{r \rightarrow 0_{+}} \frac{1}{2 r} \ell(X \cap[x-r, x+r])=1 .
$$


Lemma 12. Let $X \in \mathcal{B}(\mathbb{R})$ and $x \in X$ a Lebesgue point of $\chi_{X}$. Then

$$
\lim _{r \rightarrow 0_{+}} \frac{1}{r} \ell(X \cap[x-r, x])=1
$$

and

$$
\lim _{r \rightarrow 0_{+}} \frac{1}{r} \ell(X \cap[x, x+r])=1 .
$$

Proof. We prove the first equality; the proof of the second is similar. Let $\epsilon>0$. There is a $\delta>0$ such that $\ell(X \cap[x-r, x+r]) \geq 2 r-\epsilon r$, whenever $r<\delta$. Since $\ell(X \cap(x, x+r]) \leq r$, it follows that $\ell(X \cap[x-r, x])=\ell(X \cap[x-r, x+r])-\ell(X \cap$ $(x, x+r]) \geq 2 r-\epsilon r-r=r(1-\epsilon)$ if $0<r<\delta$.

Lemma 13. Let $f:[0, \infty) \rightarrow[0, \infty)$ be a (not necessarily strictly) decreasing function, and let $X \in \mathcal{B}(\mathbb{R})$. Suppose that there are numbers $q \in[0,1]$ and $\delta>0$ such that $\ell([0, r] \cap X) \geq r q$, whenever $0<r \leq \delta$. Then

$$
\int_{X \cap[0, \delta]} f(x) \mathrm{d} x \geq q \int_{[0, \delta]} f(x) \mathrm{d} x .
$$

Proof. By changing the values of $f$ on a countable set if necessary, we may assume that $f$ is left continuous. Denote $a=f(0)-f(\delta)$. If $n \in \mathbb{N}$, for $k=0,1, \cdots, n$ write

$$
x_{k}=\sup \left\{x \in[0, \delta] \mid f(x) \geq f(0)-\frac{k}{n} a\right\},
$$

so that $0 \leq x_{0} \leq x_{1} \leq \cdots \leq x_{n}=\delta$. If $x_{k} \leq t \leq x_{k+1}$, then

$$
f(0)-\frac{k}{n} a \geq f(t) \geq f(0)-\frac{k+1}{n} a,
$$

and it is easily seen that

$$
\delta f(\delta)+\frac{a}{n} \sum_{k=1}^{n} x_{k} \geq \int_{[0, \delta]} f(x) \mathrm{d} x \geq \delta f(\delta)+\frac{a}{n} \sum_{k=0}^{n-1} x_{k} .
$$

Since the difference of the left and right extremes is $\frac{a}{n} \delta$, we see that for any $\epsilon>0$, $n$ can be chosen such that $\frac{a}{n} \delta<\epsilon$ and thus

$$
\delta f(\delta)+\frac{a}{n} \sum_{k=0}^{n-1} x_{k}+\epsilon \geq \int_{[0, \delta]} f(x) \mathrm{d} x \geq \delta f(\delta)+\frac{a}{n} \sum_{k=0}^{n-1} x_{k} .
$$

Let $\ell_{2}$ be the two-dimensional Lebesgue measure and denote

$$
Z_{k}=\left[0, x_{k}\right] \times\left[f(0)-\frac{k+1}{n} a, f(0)-\frac{k}{n} a\right]
$$


for $k=0, \cdots, n-1$, and $Z_{n}=[0, \delta] \times[0, f(\delta)]$. Then by the assumption we get

$$
\begin{aligned}
\int_{X \cap[0, \delta]} f(x) \mathrm{d} x & \geq \sum_{k=0}^{n} \ell_{2}\left(Z_{k} \cap\{(x, y) \mid x \in X\}\right) \\
& =f(\delta) \ell([0, \delta] \cap X)+\frac{a}{n} \sum_{k=0}^{n-1} \ell\left(\left[0, x_{k}\right] \cap X\right) \\
& \geq q\left[\delta f(\delta)+\frac{a}{n} \sum_{k=0}^{n-1} x_{k}\right] \geq q \int_{[0, \delta]} f(x) \mathrm{d} x-q \epsilon .
\end{aligned}
$$

Letting $\epsilon \rightarrow 0$ we get the claim.

Theorem 14. Let $\left(f_{n}\right)_{n \in \mathbb{N}}$ be a sequence of functions $f_{n}: \mathbb{R} \rightarrow[0, \infty)$ such that

(i) $f_{n}(x) \leq f_{n}(y)$, if $x \leq y \leq 0$;

(ii) $f_{n}(x) \geq f_{n}(y)$, if $0 \leq x \leq y$;

(iii) $\int_{\mathbb{R}} f_{n}(x) \mathrm{d} x=1$;

(iv) $\lim _{n \rightarrow \infty} \int_{[-\delta, \delta]} f_{n}(x) \mathrm{d} x=1$ for any $\delta>0$.

(a) If $X \in \mathcal{B}(\mathbb{R})$ is such that $0 \in X$ and 0 is a Lebesgue point of $\chi_{X}$, then $\lim _{n \rightarrow \infty} \int_{X} f_{n}(x) \mathrm{d} x=1$.

(b) If $X \in \mathcal{B}(\mathbb{R})$ is such that $\ell(X)>0$, then there is a point $a \in X$ such that defining $g_{n}(x)=f_{n}(x-a)$ we have $\lim _{n \rightarrow \infty} \int_{X} g_{n}(x) \mathrm{d} x=1$.

Proof. (a) By Lemma 12 we may choose $\delta>0$ such that $\ell([0, r] \cap X)>r(1-\epsilon)$ and $\ell([-r, 0) \cap X)>r(1-\epsilon)$ whenever $0<r \leq \delta$. By Lemma 13 we then have $\int_{X \cap[0, \delta]} f_{n}(x) \mathrm{d} x \geq(1-\epsilon) \int_{[0, \delta]} f_{n}(x) \mathrm{d} x$, and by an analogous argument we also get $\int_{X \cap[-\delta, 0)} f_{n}(x) \mathrm{d} x \geq(1-\epsilon) \int_{[-\delta, 0)} f_{n}(x) \mathrm{d} x$. Since $\lim _{n \rightarrow \infty} \int_{[-\delta, \delta]} f_{n}(x) \mathrm{d} x=1$, there is $n_{0} \in \mathbb{N}$ such that $\int_{[-\delta, \delta]} f_{n}(x) \mathrm{d} x>1-\epsilon$ whenever $n \geq n_{0}$. Thus

$$
\begin{aligned}
\int_{X} f_{n}(x) \mathrm{d} x & \geq \int_{X \cap[-\delta, 0)} f_{n}(x) \mathrm{d} x+\int_{X \cap[0, \delta]} f_{n}(x) \mathrm{d} x \\
& \geq(1-\epsilon)\left[\int_{[-\delta, 0)} f_{n}(x) \mathrm{d} x+\int_{[0, \delta]} f_{n}(x) \mathrm{d} x\right] \geq(1-\epsilon)^{2}
\end{aligned}
$$

for all $n \geq n_{0}$.

(b) The Lebesgue points of $\chi_{X}$ form a set whose complement has measure zero (see [16, Theorem 7.7, p. 138]). Since $\ell(X)>0$, there is such a point $a \in X$. Using a translation, we may reduce the proof of this part to (a).

Remark 15. In proving part (a) above the monotonicity conditions (i) and (ii) cannot be dispensed with. For example, let

$$
X=\{0\} \cup \bigcup_{n=1}^{\infty}\left(\left[-\frac{1}{2^{n}},-\frac{1}{2^{n+1}}\left(1+\frac{1}{n}\right)\right] \cup\left[\frac{1}{2^{n+1}}\left(1+\frac{1}{n}\right), \frac{1}{2^{n}}\right]\right) .
$$

It is easy to show that 0 is a Lebesgue point of $\chi_{X}$. On the other hand, we can find (even continuous) functions $f_{n}: \mathbb{R} \rightarrow[0, \infty)$ such that $\int_{\mathbb{R}} f_{n}(x) \mathrm{d} x=1$ and the 
support of $f_{n}$ is contained in the open interval $\left(\frac{1}{2^{n+1}}, \frac{1}{2^{n+1}}\left(1+\frac{1}{n}\right)\right)$, so that (iv) holds but $\int_{X} f_{n}(x) \mathrm{d} x=0$ for all $n \in \mathbb{N}$.

We are now ready to derive a sufficient condition for a phase observable $E$ to satisfy $\|E(X)\|=1$ whenever $E(X) \neq O$. Let $E$ be given with its phase matrix $\left(c_{n, m}\right)_{n, m \in \mathbb{N}}$. For any unit vector $\psi \in \mathcal{H}$, the phase probability measure $p_{\psi}^{E}$ is absolutely continuous with respect to the Lebesgue measure $\ell$. Let $g_{\psi}^{E}$ denote the Radon-Nikodým derivative, so that $p_{\psi}^{E}(X)=(2 \pi)^{-1} \int_{X} g_{\psi}^{E}(\theta) \mathrm{d} \theta$. This is a $2 \pi$ periodic density function $\mathbb{R} \rightarrow[0, \infty]$. Consider the following class of unit vectors

$$
\psi_{r}:=\sqrt{1-r^{2}} \sum_{n=0}^{\infty} e^{i v_{n}} r^{n}|n\rangle, \quad r \in(-1,1),
$$

where $\left(v_{n}\right)_{n \in \mathbb{N}} \subset \mathbb{R}$. The density function $g_{\psi_{r}}^{E}$ is continuous and of the form

$$
g_{\psi_{r}}^{E}(\theta)=\left(1-r^{2}\right) \sum_{n, m=0}^{\infty} c_{n, m} e^{-i\left(v_{n}-v_{m}\right)} r^{n+m} e^{i(n-m) \theta},
$$

where the series converges absolutely.

Lemma 16. With the above notations, if $\lim _{n \rightarrow \infty} c_{n, n+k} e^{-i\left(v_{n}-v_{n+k}\right)}=1$ for all $k \in$ $\mathbb{N}$, for some $\left(v_{n}\right)_{n \in \mathbb{N}} \subset \mathbb{R}$, then

$$
\lim _{r \rightarrow 1-} \frac{1}{2 \pi} \int_{[-\delta, \delta]} g_{\psi_{r}}^{E}(\theta) \mathrm{d} \theta=1
$$

for all $\delta>0$.

Proof. Suppose that

$$
\lim _{n \rightarrow \infty} c_{n, n+k} e^{-i\left(v_{n}-v_{n+k}\right)}=1
$$

for all $k \in \mathbb{Z}^{+}$, where $\left(v_{n}\right)_{n \in \mathbb{N}} \subset \mathbb{R}$. The Fourier-Stieltjes coefficients of the probability measure $p_{\psi_{r}}^{E}$ are of the form

$$
c_{k}^{r}:=\frac{1}{2 \pi} \int_{0}^{2 \pi} e^{-i k \theta} g_{\psi_{r}}^{E}(\theta) \mathrm{d} \theta=r^{k}\left(1-r^{2}\right) \sum_{n=0}^{\infty} c_{n+k, n} e^{-i\left(v_{n+k}-v_{n}\right)} r^{2 n}
$$

and $c_{-k}^{r}=\overline{c_{k}^{r}}$ for all $k \in \mathbb{N}$ and $r \in(-1,1)$. Next we show that $\lim _{r \rightarrow 1-} c_{k}^{r}=1$ for all $k \in \mathbb{Z}$.

Fix $k \in \mathbb{Z}^{+}$and $\epsilon>0$. Since (25) holds, one may choose such an $n_{\epsilon} \in \mathbb{Z}^{+}$ that $\left|c_{n+k, n} e^{-i\left(v_{n+k}-v_{n}\right)}-1\right|<\epsilon / 2$ for all $n \geq n_{\epsilon}$. Since $\sum_{n=0}^{\infty} r^{2 n}=1 /\left(1-r^{2}\right)$, $r \in(-1,1)$, one gets

$$
\begin{aligned}
\left|c_{k}^{r} / r^{k}-1\right| \leq & \left(1-r^{2}\right) \sum_{n=0}^{n_{\epsilon}-1}\left|c_{n+k, n} e^{-i\left(v_{n+k}-v_{n}\right)}-1\right| r^{2 n} \\
& +\left(1-r^{2}\right) \sum_{n=n_{\epsilon}}^{\infty}\left|c_{n+k, n} e^{-i\left(v_{n+k}-v_{n}\right)}-1\right| r^{2 n} \\
\leq & 2\left(1-r^{2 n_{\epsilon}}\right)+\epsilon / 2 .
\end{aligned}
$$


Choose $r_{\epsilon} \in[0,1)$ such that $2\left(1-r^{2 n_{\epsilon}}\right)<\epsilon / 2$ when $r \in\left[r_{\epsilon}, 1\right)$ to get $\left|c_{k}^{r} / r^{k}-1\right|<\epsilon$ for all $r \in\left[r_{\epsilon}, 1\right)$. Thus, $c_{k}^{r} \sim r^{|k|}$ and $c_{k}^{r} \rightarrow 1$ for all $k \in \mathbb{Z}$ when $r \rightarrow 1-$.

The condition $\lim _{r \rightarrow 1-} c_{k}^{r}=1, k \in \mathbb{Z}$, implies that

$$
\lim _{r \rightarrow 1-} \frac{1}{2 \pi} \int_{-\delta}^{\delta} g_{\psi_{r}}^{E}(\theta) \mathrm{d} \theta=1
$$

for all $\delta>0$.

Lemma 16 applies, in particular, to the canonical phase. Moreover, in that case the density function $g_{\psi_{r}}^{E_{\mathrm{ca}}}$ is simply

$$
g_{\psi_{r}}^{E_{\mathrm{can}}}(\theta)=\left(1-r^{2}\right) \sum_{n, m=0}^{\infty} r^{n+m} e^{i(n-m) \theta}=\frac{1-r^{2}}{1-2 r \cos \theta+r^{2}} .
$$

Defining $f_{n}(x):=g_{\psi_{1-(n+1)-1}}^{E_{\text {can }}}(x),|x| \leq \pi$, and $f_{n}(x)=0,|x|>\pi$, one gets a sequence $\left(f_{n}\right)_{n \in \mathbb{N}}$ which fulfills the conditions of Theorem 14. Consider next the phase observable $E_{|0\rangle}$. For coherent states $|r\rangle=e^{-r^{2} / 2} \sum_{n \geq 0} \frac{r^{n}}{\sqrt{n}}|n\rangle, r \geq 0$, we obtain [5]

$$
g_{|r\rangle}^{E_{|0\rangle}}(\theta)=\int_{r^{2}}^{\infty} e^{-v} \mathrm{~d} v+e^{-r^{2} \sin ^{2} \theta} 2 r \cos \theta \int_{-r \cos \theta}^{\infty} e^{-u^{2}} \mathrm{~d} u .
$$

Functions $f_{n}(x):=g_{|r=n\rangle}^{E_{|0\rangle}}(x),|x| \leq \pi, f_{n}(x)=0,|x|>\pi$, also fulfill the conditions of Theorem 14. Hence we have the following results:

Proposition 17. If $X \in \mathcal{B}([0,2 \pi))$ has nonzero Lebesgue measure, then $\left\|E_{\text {can }}(X)\right\|=$ 1 and $\left\|E_{|0\rangle}(X)\right\|=1$.

Hence both the canonical phase $E_{\text {can }}$ and the ground state phase $E_{|0\rangle}$ fulfill this necessary condition for the number-phase complementarity. The question remains, however, whether these observables actually are complementary to the number.

\section{ON NUMBER-PHASE UNCERTAINTY RELATIONS}

The number-phase uncertainty relations are often presented as a kind of quantitative expression for the complementarity of this pair of observables. Although we do not support this viewpoint, we find it useful to briefly elaborate on the number-phase uncertainty product, especially for high amplitude coherent states.

A phase observable $E$ is a periodic quantity. Therefore, the variance $\operatorname{Var}(E, \psi)$ of the phase distribution $p_{\psi}^{E}$ in a vector state $\psi \in \mathcal{H},\|\psi\|=1$, though well defined, is not a good measure of phase uncertainty. For periodic distributions the appropriate notion is that of minimun variance, introduced by Lévy [17]. Using the density $g_{\psi}^{E}$ of $p_{\psi}^{E}$, the minimum variance of the phase distribution $p_{\psi}^{E}$ is then defined as

$$
\operatorname{VAR}(E, \psi):=\inf \left\{\frac{1}{2 \pi} \int_{\beta-\pi}^{\beta+\pi}(\theta-\alpha)^{2} g_{\psi}^{E}(\theta) d \theta \mid \alpha, \beta \in \mathbb{R}\right\},
$$


and one finds that $0 \leq \operatorname{VAR}(E, \psi) \leq \pi^{2} / 3$. The minimum variance of the canonical phase $E_{\text {can }}$ in a coherent state $|z\rangle$ has, for large $|z|$, the following asymptotic form (for details, see [5, VII A,C]):

$$
\operatorname{VAR}\left(E_{\mathrm{can}},|z\rangle\right) \simeq \frac{1}{4} \frac{1}{|z|^{2}}
$$

On the other hand, the variance of the number observable $N$ in a coherent state $|z\rangle$ is $\operatorname{Var}(N,|z\rangle)=|z|^{2}$. so that, for large $|z|$, one has

$$
\operatorname{Var}(N,|z\rangle) \operatorname{VAR}\left(E_{\text {can }},|z\rangle\right) \simeq \frac{1}{4}
$$

The logical independence of complementarity and uncertainty relations in general has been clearly established long ago $[18$. The concept of complementarity is linked with the impossibility of joint measurements of two observables. By contrast, as is evident from the above formalisations, the uncertainty relation, as well as the notions of probabilistic and value complementarity, refer to features of the probability distributions of separate, independent measurements of the two observables in question.

\section{Conclusion}

The set of covariant phase observables is a convex set in a natural way. Let $E$ be any phase observable. Then $E_{\epsilon}(X)=\epsilon E_{\text {triv }}(X)+(1-\epsilon) E(X), X \in \mathcal{B}([0,2 \pi))$, defines a phase observable which is not complementary with number. Indeed, $E_{\epsilon}(X) \geq \epsilon P_{k}$ for all $k \geq 0$ so that $E_{\epsilon}(X) \wedge P_{k} \geq \epsilon P_{k}$ for all $k \geq 0$. This shows that every phase observable $E$ is "arbitrarily close" to a phase observable $E_{\epsilon}$ which is not complementary with the number.

This observation, which generalises to every canonical pair, implies that the complementarity of such pairs, given that it holds, is not strictly testable. This is also true for probabilistic complementarity in the sense that finite statistics can never confirm strictly whether a given event has probability equal to one. Nevertheless, complementarity indicates a relation between two observables which is robust under small imprecisions: if a pair of observables is complementary, then a "nearby" noncomplementary pair will only allow "small" positive joint lower bounds between their positive operators.

The canonical phase, as well as any unitarily equivalent one, is an extremal element of the convex set of phase observables. Indeed, by Theorem 1, for all such phase observables $\left|c_{n, m}\right|=1, n, m \geq 0$. Let $\left(c_{n, m}\right)$ be the phase matrix of a phase observable $E$, and assume that $E$ is a convex combination of phase observables $E_{1}$ and $E_{2}$, that is, $c_{n, m}=\lambda c_{n, m}^{(1)}+(1-\lambda) c_{n, m}^{(2)}$, with some $0<\lambda<1$. If $\left|c_{n, m}\right|=1$, it follows that $\left|c_{n, m}^{(1)}\right|=\left|c_{n, m}^{(2)}\right|=1$, and that the phases of $c_{n, m}^{(1)}$ and $c_{n, m}^{(2)}$ are the same. Therefore, $c_{n, m}=c_{n, m}^{(1)}=c_{n, m}^{(2)}$ for all $n, m \geq 0$, that is, $E=E_{1}=E_{2}$. We conclude with the conjecture that further analysis of the convex structure of the set of phase observables may help to decide on the open question of the existence of a phase that is complementary to number. 


\section{REFERENCES}

[1] A.S. Holevo, Generalized imprimitivity systems for abelian groups, Izvestiya VUZ, Matematika 27 (1983) 49-71. English translation, Soviet Math., 53-80.

[2] P. Lahti, J.-P. Pellonpää, Covariant phase observables in quantum mechanics, Journal of Mathematical Physics 40 (1999) 4688-4698.

[3] G. Cassinelli, E. De Vito, P. Lahti, J.-P. Pellonpää, Covariant localizations in the torus and the phase observables, manuscript to be submitted.

[4] A.S. Holevo, Probabilistic and Statistical Aspect of Quantum Theory, North-Holland Publ. Co., Amsterdam, 1982.

[5] P. Lahti, J.-P. Pellonpää, Characterizations of the canonical phase observable, Journal of Mathematical Physics 41 (2000) 7352-7381.

[6] G. Ludwig, Foundations of Quantum Mechanics, Vol I, Springer-Verlag, Berlin, 1983.

[7] K. Kraus, States, Effects, and Operations LNP 190, Springer, Berlin, 1983.

[8] P. Busch, P. Lahti, P. Mittelstaedt, The Quantum Theory of Measurement, LNP m2, Springer-Verlag, Berlin, 2nd revised edition, 1996.

[9] P. Lahti, S. Pulmannova, K. Ylinen, Coexistent observables and effects in a convexity approach, Journal of Mathematical Physics 39 (1998) 6364-6371.

[10] C. Berg, J.P.R. Christensen, P. Ressel, Harmonic Analysis on Semigroups, SpringerVerlag, New York, 1984.

[11] K. Ylinen, Positive operator bimeasures and a noncommutative generalization, Studia Mathematica 118 (1996) 157-168.

[12] N. Bohr, The quantum postulate and the recent development of atomic theory, $\mathrm{Na}$ ture 121 (1928) 580-590. Can quantum mechanical description of physical reality be considered complete? Physical Review 48 (1935) 696-702.

[13] P. Busch, S. Gudder, Effects as functions on projective Hilbert space, Letters in Mathematical Physics 47 (1999) 329-337.

[14] N. Young, An introduction to Hilbert space, Cambridge University Press, Cambridge, 1988.

[15] P. Billingsley, Probability and Measure, John Wiley \& Sons, Inc., New York, 1979.

[16] W. Rudin, Real and Complex Analysis, McGraw-Hill, 1987 e/3.

[17] P. Lévy, L'addition des variables aléatoires définies sur une circonférence, Bull. Soc. Math. France 67, 1-41 (1939).

[18] P.J. Lahti, On the inter-relations of the three quantal principles, Current Issues in Quantum Logic, eds Enrico B. Beltrametti and Bas C. van Fraassen, Plenum Publishing Corporation, New York, 1981, pp 447-454.

Paul Busch, Department of Mathematics, University of Hull, Hull, England

E-mail address: p.busch@maths.hull.ac.uk

Pekka Lahti, Department of Physics, University of Turku, Fin-20014 Turku, FINLAND

E-mail address: pekka.lahti@utu.fi

Juha-Pekka Pellonpä̈̈, Department of Physics, University of Turku, Fin-20014 TuRKu, Finland

E-mail address: juhpello@utu.fi

Kari Ylinen, Department of Mathematics, University of Turku, Fin-20014 Turku, FINLAND

E-mail address: kari.ylinen@utu.fi 